\title{
Massive binaries and the enrichment of the interstellar medium in globular clusters
}

\author{
S. E. de Mink, ${ }^{1}$ O. R. Pols, ${ }^{1}$ N. Langer ${ }^{2,1}$ and R. G. Izzard ${ }^{3}$ \\ ${ }^{1}$ Astronomical Institute Utrecht, Postbus 80000, 3508 TA Utrecht, The Netherlands \\ ${ }^{2}$ Argelander-Institut für Astronomie, Auf dem Hügel 71, 53121 Bonn, Germany \\ ${ }^{3}$ Université Libre de Bruxelles, Boulevard du Triomphe, B-1050 Brussels, Belgium \\ email: S.E.deMink@uu.nl
}

\begin{abstract}
Abundance anomalies observed in globular cluster stars indicate pollution with material processed by hydrogen burning. Two main sources have been suggested: asymptotic giant branch (AGB) stars and massive stars rotating near the break-up limit (spin stars). We discuss the idea that massive binaries may provide an interesting alternative source of processed material. We discuss observational evidence for mass shedding from interacting binaries. In contrast to the fast, radiatively driven winds of massive stars, this material is typically ejected with low velocity. We expect that it remains inside the potential well of a globular cluster and becomes available for the formation or pollution of a second generation of stars. We estimate that the amount of processed low-velocity material that can be ejected by massive binaries is larger than the contribution of the two previously suggested sources combined.
\end{abstract}

Keywords. globular clusters, binaries: close, stars: abundances, ISM: jets and outflows

\section{Introduction}

For a long time, star clusters have been considered idealized, single-age, chemically homogeneous stellar populations. However, it has recently become clear that some clusters show multiple main sequences and subgiant branches, and extended horizontal branches (e.g., Piotto et al. 2007). The presence of these features in some intermediate-age clusters may be explained by the effects of stellar rotation on the location of stars in the colourmagnitude diagram (Bastian \& de Mink 2009). However, this provides no explanation for the features seen in old globular clusters, which seems to imply the existence of multiple populations within one cluster.

In addition, large star-to-star abundance variations are found for light elements such as $\mathrm{C}, \mathrm{N}, \mathrm{O}, \mathrm{Na}$ and $\mathrm{Al}$, while the composition of heavier elements (Fe-group and $\alpha$ elements) seems to be constant. Field stars with the same metallicity do not exhibit these abundance patterns (for a review see Gratton et al. 2004). These chemical variations have been interpreted as originating from the presence of both a 'normal' stellar population, exhibiting abundances similar to field stars of the same metallicity, and a second population of stars formed from material processed by hydrogen burning via the CNO cycle and the $\mathrm{NeNa}$ and $\mathrm{MgAl}$ chains (e.g., Prantzos et al. 2007). According to Carretta et al. (2009), 50-70\% of the stars in gloular clusters belong to the second population.

Two sources of processed ejecta have been proposed: the slow winds of massive $A G B$ stars, which enrich their convective envelopes with H-burning products (Ventura et al. 2001; Dantona et al. 2002; Denissenkov \& Herwig 2003) and rapidly rotating massive stars (which we refer to as spin stars), which are believed to expel processed material centrifugally when they reach break-up rotation (Prantzos \& Charbonnel 2006; Decressin 


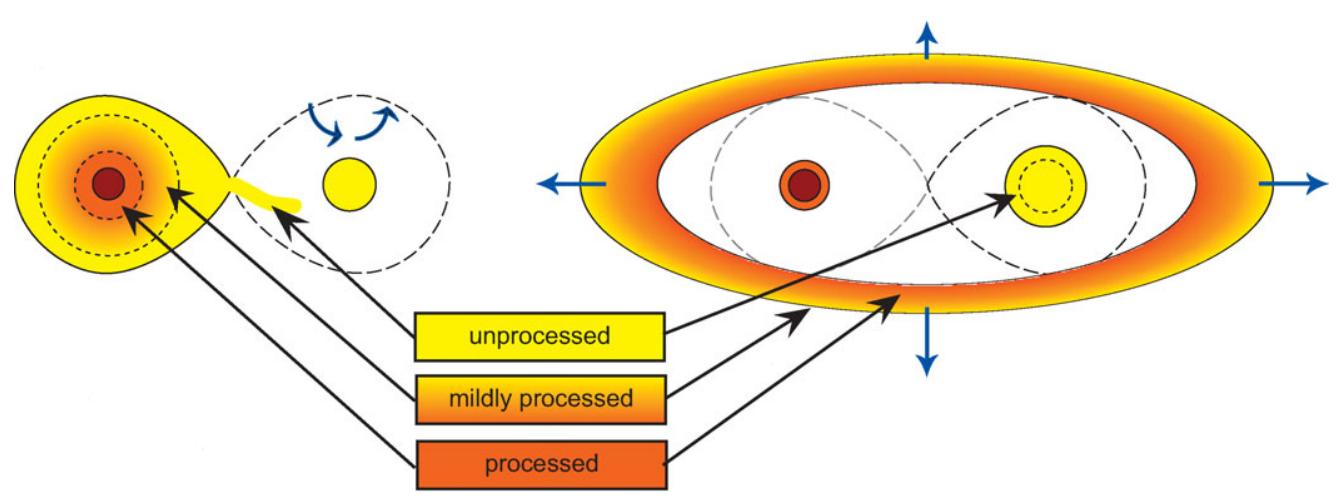

Figure 1. Cartoon representation of the proposed scenario. (left) A binary system at the onset of mass transfer. The deepest layers in the donor star have been processed by proton-capture reactions. The accreting star spins up as it accretes mass and angular momentum until it approaches the break-up limit. (right) The same system after the donor star has been stripped of its envelope. The companion star accreted just a fraction of the transferred mass, mainly unprocessed material originating from the outermost layers of the donor star. Material orginating from deeper layers in the donor star is shed into a circumbinary disk.

et al. 2007a). In this scenario a first generation of stars is formed from pristine material. Their low-velocity ejecta are trapped inside the potential well of the cluster and provide the material for the formation of a second generation of stars. $\dagger$

Although both proposed sources are promising, matching the observed abundance patterns and providing enough ejecta for the formation of a second generation which outnumbers the first have proven to be two major challenges. Here, we propose massive binaries as a candidate for the internal pollution of globular clusters.

\section{Binaries as sources of enrichment}

Interacting binaries can shed large amounts of material processed by hydrogen burning into their surroundings. A clear example is the well-studied system RY Scuti. It is undergoing rapid mass transfer from a $7 \mathrm{M}_{\odot}$ supergiant to its $30 \mathrm{M}_{\odot}$ companion. Mass is lost from the system via the outer Lagrangian points into a circumbinary disk and a wider double-toroidal nebula. The nebula shows signatures of CNO processing: it is enriched in helium and nitrogen and depleted in oxygen and carbon (Smith et al. 2002; Grundstrom et al. 2007). In contrast to the high-velocity radiatively driven winds of massive stars, these ejecta have low velocities. Smith et al. (2001) measure expansion velocities ranging from 30 to $70 \mathrm{~km} \mathrm{~s}^{-1}$ in the nebula of RY Scuti, which are smaller than the present-day escape velocity of massive globular clusters. Furthermore, the nebula shows evidence for clumps (Smith et al. 2002) and dust in the outer parts (Gehrz et al. 2001), which may serve as seeds for the formation of a second generation of low-mass protostars.

Evidence for severe mass loss from interacting binaries comes from a wide variety of observed interacting and post-interaction systems: it appears to be a common phenomenon for many interacting binaries. Various authors have inferred highly nonconservative evolution for Algols, systems which are currently undergoing stable mass transfer (e.g., Refsdal et al. 1974; de Greve \& Linnell 1994; Figueiredo et al. 1994; van Rensbergen et al. 2006;

$\dagger$ Glebbeek et al. (2009) suggested that a chain of multiple stellar collisions in the dense centre of a star cluster may also enrich the interstellar medium with processed material. 
de Mink et al. 2007). Most notable are short-period binaries containing a compact object, e.g., cataclysmic variables, X-ray binaries, binary radio pulsars and double white-dwarf systems. Their formation requires a phase of severe mass and angular-momentum loss through ejection of a common envelope. Direct evidence for this type of evolution comes from planetary nebulae with close binary nuclei, which appear to have recently emerged from the common-envelope phase (for a review see Iben \& Livio 1993).

Theoretical considerations support the idea that most interacting binaries shed large amounts of mass. Three-dimensional hydrodynamical simulations of the mass-transfer stream and accretion disk of the interacting binary $\beta$ Lyrae predict that $50 \%$ of the transferred mass is lost (Bisikalo et al. 2000; Nazarenko \& Glazunova 2006). In addition, Ulrich \& Burger (1976) showed that the accreting star is driven out of thermal equilibrium and expands. This can lead to contact and strong mass and angular-momentum loss from the system (Flannery \& Ulrich 1977). Furthermore, Packet (1981) noted that the accreting star reaches break-up rotation after gaining only a few percent of its own mass. Rapid rotation is found for many accreting stars in Algols (Barai et al. 2004) and this mechanism has been proposed to explain the formation of Be X-ray binaries (e.g., Pols et al. 1991). In principle, tides can counteract the effect of spin-up through mass transfer in close binaries. Petrovic et al. (2005) computed detailed binary evolution models, taking into account these effects. They find that massive binaries with initial periods as short as 3-6 days lose $70-80 \%$ of the transferred mass, on average. For wider and more massive systems, they expect even less conservative mass transfer, such that nearly the entire envelope of the primary is returned to the interstellar medium.

\section{Composition of the ejecta}

In de Mink et al. (2009b) we present the computations of the evolution of a $20 \mathrm{M}_{\odot}$ star in a close binary, taking into account the effects of nonconservative mass and angularmomentum transfer, rotation and tidal interaction. We find that this system sheds about $10 \mathrm{M}_{\odot}$ of material, nearly the entire envelope of the primary star. The ejecta are enriched in $\mathrm{He}, \mathrm{N}, \mathrm{Na}$ and $\mathrm{Al}$, and depleted in $\mathrm{C}$ and $\mathrm{O}$, similar to the abundance patterns observed in gobular cluster stars. However, $\mathrm{Mg}$ is not significantly depleted in the ejecta of this model. For a more detailed discussion, we refer to de Mink et al. (2009b).

\section{Mass budget}

One of the main challenges for the two previously proposed sources of pollution, massive AGB and spin stars, is to provide the large amount of ejecta needed to create a second population which is larger than the first. The population of low-mass stars $\left(0.1-0.8 \mathrm{M}_{\odot}\right)$, which can still be observed today, represents $38 \%$ of the stellar mass initially present in the cluster, assuming a standard Kroupa (2001) initial mass function (IMF) between 0.1 and $120 \mathrm{M}_{\odot}$ (see Figure 2). The ejecta of AGB stars with initial masses between 4 and $9 \mathrm{M}_{\odot}$ represent up to $8.9 \%$ of the initial stellar mass (assuming an initial-final-mass relation as given by Ciotti et al. 1991). For spin stars, this fraction is $3.4 \%$ if one assumes that every massive star is single and born with a rotational velocity high enough to reach break-up rotation (using the models of Decressin et al. 2007a). These ejecta are not sufficient to create a second generation which is equally numerous as the first, even when we assume that the second generation consists only of low-mass stars and that star formation is very efficient (see Figure 2).

Two rather extreme solutions have been proposed. 
(1) The IMF was highly anomalous, favouring the formation of the polluting stars with respect to the long-lived low-mass stars that we observe today. Even though we have no direct constraints on the IMF of globular clusters, Kroupa (2002) finds that the IMF is remarkably uniform in stellar populations with very different properties.

(2) Clusters were initially at least 10-20 times more massive and they have preferentially lost low-mass stars from the first generation as a result of dynamical evolution and tidal stripping (Decressin et al. 2007b, 2008; D'Ercole et al. 2008). In this section we investigate to what extent the ejecta of massive binaries can alleviate this conundrum.

Even though the current fraction of detected binaries in globular clusters is not high (e.g., Davies et al. 2008), this is not necessarily the case for the high-mass stars originally present in the cluster. Sana et al. (2008) and Mason et al. (2009) find a minimum binary fraction of $60-75 \%$ for $\mathrm{O}$ stars associated with clusters or OB associations. In globular clusters, these fractions may even be higher. In this environment, close binaries can be created and tightened during and after the star-formation process, for example by the dissipative interaction with gas (e.g., Bonnell \& Bate 2005) and by three-body interactions such as the Kozai mechanism in combination with tidal friction (Fabrycky et al. 2007). With massive stars preferentially residing in the dense core of the cluster, where the dynamical encounters are most frequent, it is not unreasonable to assume that the large majority of massive stars interact through mass exchange.

Let us assume that every massive star is a member of an interacting binary. In Section 2 we argued that nearly the entire envelope of the donor is returned to the interstellar medium. For simplicity, we neglect the contribution of the secondary star after it has been spun up by mass transfer or during a possible phase of reverse mass transfer, and we assume that entire envelope of the primary becomes available for star formation. We assume helium core masses as in Prantzos \& Charbonnel (2006) for stars more massive than $10 \mathrm{M}_{\odot}$. Under these assumptions, the slow ejecta of massive binaries represent $13 \%$

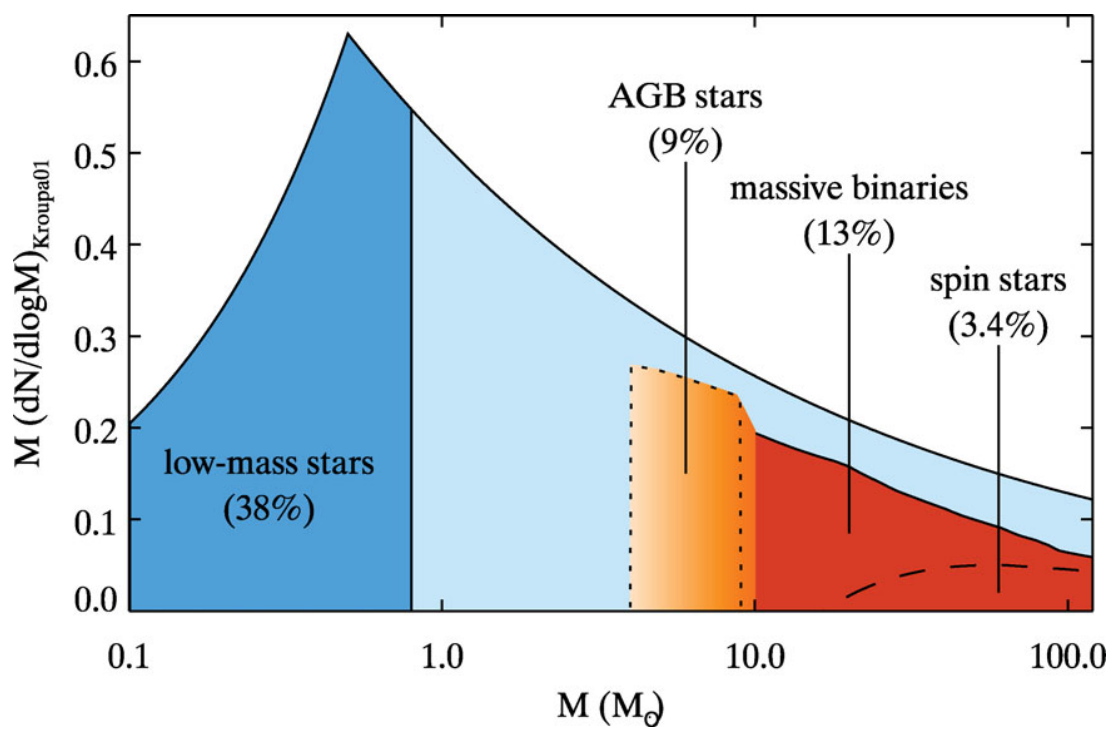

Figure 2. Mass weighted Kroupa (2001) IMF as a function of stellar mass. The surface areas indicate the mass contained in the first generation of long-lived, low-mass stars (dark blue), the ejecta of AGB stars (dotted line), spin stars, i.e., rapidly rotating massive stars (dashed line) and massive (red) and intermediate-mass (orange) binaries. Percentages indicate the fraction of mass relative to the total mass contained in stars of the first generation. See Section 4 for details. (Figure adapted from de Mink et al. 2009b.) 
of the mass originally present in stars, i.e., more than the ejecta of AGB and spin stars combined.

Measurements of lithium suggest that the ejecta of the first generation are diluted with pristine gas (Pasquini et al. 2005). Together with an equal amount of pristine gas, the ejecta of binaries with donors more massive than $10 \mathrm{M}_{\odot}$ would represent $26 \%$ of the initial cluster mass (compared to $38 \%$ contained in the first generation of low-mass stars). The adopted lower-mass limit for our binary scenario is rather arbitrary. If we take into account the potential contribution of intermediate-mass stars $\left(4-10 \mathrm{M}_{\odot}\right)$, the ejecta would be sufficient to form a second population of chemically peculiar stars that outnumbers the first generation of normal stars. The assumptions in this scenario can be relaxed if the evaporation of stars from the cluster preferentially affects the first stellar generation, as suggested by Decressin et al. (2008).

\section{Conclusions}

We discussed the potential of massive binaries as the source of enrichment in globular clusters. The majority of massive stars are expected to be members of interacting binary systems. These return most of the envelope of their primary star to the interstellar medium during nonconservative mass transfer. We show that the amount of polluted material ejected by binaries may be larger than that of the two previously suggested sources, massive AGB stars and the slow winds of rapidly rotating massive stars. After dilution with pristine material, as lithium observations suggest, binaries could return enough material for the formation of a chemically enriched second generation that is equally numerous as the first generation of low-mass stars, without the need to assume a highly anomalous IMF, external polution of the cluster or significant loss of stars from the nonenriched first generation.

In addition to providing a new source of slowly ejected enriched material, binary interactions also affect the previously proposed scenarios. Binary mass transfer naturally produces a large number of rapidly rotating massive stars which may enrich their surroundings even further. Binary interaction will also affect the yields of intermediate-mass stars. Premature ejection of the envelope in $4-9 \mathrm{M}_{\odot}$ stars will result in ejecta with less pronounced anticorrelations as suggested in the AGB scenario. On the other hand, we expect that binary-induced mass loss may also prevent the dredge up of helium-burning products.

For a detailed comparison of the chemical predictions of this scenario, binary models for a range of masses and orbital periods are needed and population synthesis models are essential to fully evaluate the mass budget of the different sources. Some peculiar features, such as the apparant presence of distinct, chemically homogeneous subpopulations in $\omega$ Cen and NGC 2808 (e.g., Renzini 2008) deserve further attention.

\section{References}

Barai, P., et al. 2004, ApJ, 608, 989

Bastian, N. \& de Mink, S. E. 2009, MNRAS (Letters), 398, L11

Bisikalo, D. V., Harmanec, P., Boyarchuk, A. A., Kuznetsov, O. A., \& Hadrava, P. 2000, A\&̊A, 353,1009

Bonnell, I. A. \& Bate, M. R. 2005, MNRAS, 362, 915

Carretta, E., Bragaglia, A., Gratton, R. G., et al. 2009, A\&A, 505, 117

Ciotti, L., D'Ercole, A., Pellegrini, S., \& Renzini, A. 1991, ApJ, 376, 380

D’Antona, F., Caloi, V., Montalbán, J., Ventura, P., \& Gratton, R. 2002, AESA, 395, 69 
Davis, D. S., Richer, H. B., Anderson, J., Brewer, J., Hurley, J., Kalirai, J. S., Rich, R. M., \& Stetson, P. B. 2008, AJ, 135, 2155

de Greve, J. P., \& Linnell, A. P. 1994, A\&A, 291, 786

de Mink, S. E., Pols, O. R., \& Hilditch, R. W. 2007, A\&\&A, 467, 1181

de Mink, S. E., Pols, O. R., Langer, N., \& Izzard, R. 2009a, A\&A, in press

de Mink, S. E., Cantiello, M., Langer, N., Pols, O. R., Brott, I., \& Yoon, S.-C. 2009b, A $6 A$, 497, 243

Decressin, T., Baumgardt, H., \& Kroupa, P. 2008, A\&A A, 492, 101

Decressin, T., Charbonnel, C., \& Meynet, G. 2007a, A\&A, 475, 859

Decressin, T., Meynet, G., Charbonnel, C., Prantzos, N., \& Ekström, S. 2007b, A\&A, 464, 1029

Denissenkov, P. A. \& Herwig, F. 2003, ApJ (Letters), 590, L99

D'Ercole, A., Vesperini, E., D'Antona, F., McMillan, S. L. W., \& Recchi, S. 2008, MNRAS, 391, 825

Fabrycky, D. \& Tremaine, S. 2007, ApJ, 669, 1298

Figueiredo, J., de Greve, J. P., \& Hilditch, R. W. 1994, A\&A, 283, 144

Flannery, B. P. \& Ulrich, R. K. 1977, ApJ, 212, 533

Gehrz, R. D., Smith, N., Jones, B., Puetter, R., \& Yahil, A. 2001, ApJ, 559, 395

Glebbeek, E., Gaburov, E., de Mink, S. E., Pols, O. R., \& Portegies Zwart, S. F. 2009, A\&A, 497,255

Gratton, R., Sneden, C., \& Carretta, E. 2004, ARA\&A, 42, 385

Grundstrom, E. D., Gies, D. R., Hillwig, T. C., McSwain, M. V., Smith, N., Gehrz, R. D., Stahl, O., \& Kaufer, A. 2007, ApJ, 667, 505

Iben, I. J. \& Livio, M. 1993, PASP, 105, 1373

Kroupa, P. 2001, MNRAS, 322, 231

Kroupa, P. 2002, Science, 295, 82

Mason, B. D., Hartkopf, W. I., Gies, D. R., Henry, T. J., \& Helsel, J. W. 2009, AJ, 137, 3358

Nazarenko, V. V. \& Glazunova, L. V. 2006, Ast. Rep., 50, 380

Packet, W. 1981, A\&SA, 102, 17

Pasquini, L., Bonifacio, P., Molaro, P., Francois, P., Spite, F., Gratton, R. G., Carretta, E., \& Wolff, B. 2005, A\& $A, 441,549$

Petrovic, J., Langer, N., \& van der Hucht, K. A. 2005, A\&SA, 435, 1013

Piotto, G., Bedin, L. R., Anderson, J., King, I. R., Cassisi, S., Milone, A. P., Villanova, S., Pietrinferni, A., \& Renzini, A. 2007, ApJ (Letters), 661, L53

Pols, O. R., Cote, J., Waters, L. B. F. M., \& Heise, J. 1991, A\& A, 241, 419

Prantzos, N. \& Charbonnel, C. 2006, A\& $A, 458,135$

Prantzos, N., Charbonnel, C., \& Iliadis, C. 2007, $A \mathscr{E} A$, 470, 179

Refsdal, S., Roth, M. L., \& Weigert, A. 1974, A\&A, 36, 113

Renzini, A. 2008, MNRAS, 391, 354

Sana, H., Gosset, E., Nazé, Y., Rauw, G., \& Linder, N. 2008, MNRAS, 386, 447

Smith, N., Gehrz, R. D., \& Goss, W. M. 2001, AJ, 122, 2700

Smith, N., Gehrz, R. D., Stahl, O., Balick, B., \& Kaufer, A. 2002, ApJ, 578, 464

Ulrich, R. K. \& Burger, H. L. 1976, ApJ, 206, 509

van Rensbergen, W., de Loore, C., \& Jansen, K. 2006, A\& $A$, 446, 1071

Ventura, P., D'Antona, F., Mazzitelli, I., \& Gratton, R. 2001, ApJ (Letters), 550, L65 